\title{
Uterine submucosal leiomyomas: modern diagnosis and miniinvasive surgery
}

\author{
Oana Denisa Balalau ${ }^{1,2^{*}}$, Mihai-George $\operatorname{Loghin}^{1,2}$, Sabin Vasilache ${ }^{1}$, Octavian Gabriel \\ Olaru $^{1,2}$, Ioana Paunica ${ }^{1}$, Ileana Maria Conea ${ }^{1,2}$ \\ ${ }^{1}$ CAROL DAVILA UNIVERSITY OF MEDICINE AND PHARMACY, FACULTY OF GENERAL MEDICINE, BUCHAREST, ROMANIA \\ ${ }^{2}$ ST. JOHN CLINICAL EMERGENCY HOSPITAL BUCHAREST, BUCUR MATERNITY, BUCHAREST, ROMANIA
}

Uterine leiomyomatosis is one of the most common benign pelvic tumors diagnosed in women aged 25-44 years. Clinically, it is manifested by vaginal bleeding, pelvic pain, infertility, digestive and urinary symptoms. The diagnosis of uterine fibroids requires careful clinical and paraclinical evaluation. Based on these data, the therapeutic decision is conducted in most cases. The treatment of uterine leiomyomatosis involves several procedures, such as: total abdominal hystectomy, total vaginal hystectomy, abdominal myomectomy, vaginal, laparoscopic or hysteroscopic myomectomy. Hysteroscopic myomectomy is currently the preferred procedure for submucosal fibroids. It has multiple advantages: shorter recovery time, reduced pain related to movements, shorter duration procedure and fewer risks. The procedure has few contraindications. The most common complication is recurrence. The treatment of choice for prolapsed pedunculated submucous leiomyoma is vaginal myomectomy. As described, the procedure has multiple advantages and generally has a low recurrence rate.
\end{abstract}

Category: Review

Received: March 14, 2021

Accepted: June 19, 2021

Published: November 15, 2021

Keywords:

uterine, submucosal leiomyomas, pelvic ultrasound, hysteroscopic myomectomy

*Corresponding author:

Oana Denisa Balalau,

Carol Davila University of Medicine and Pharmacy, St. John Clinical Emergency Hospital, Bucur Maternity, Bucharest, Romania

E-mail: doctor.balalau@gmail.com

\section{Introduction}

The uterine fibroids also known as leiomyomas or simply myomas are the most widely encountered benign monoclonal tumors in the female pelvic area $[1,2]$. Uterine fibroids develop from the myometrium, more exactly from the smooth muscle cells and fibroblasts found in the uterine structure. Leiomyomas are typically found in females that are at a reproductive age and the most representative symptoms are represented by abnormal uterine bleeding and pelvic pain or pressure.

Terminology and location of uterine fibroids depend by their location within the uterine wall, being closely related to the structures they affect. The International Federation of Gynecology and Obstetrics offers a more detailed description of all leiomyomas that concern the uterus [3], as described below.

Type 0,1 and 2 are the submucosal leiomyomas, which develop from the myometrial smooth muscle cells exactly underneath the endometrium. They usually grow and protrude inside the uterine cavity and the FIGO classification is very relevant to the physician because it foresees the outcome of the myomectomy.

Type 0 of leiomyoma is a fibroid that's completely inside the endometrial cavity.

Type 1 of leiomyoma is a fibroid with less than $50 \%$ extension of its mass in the myometrium.

Type 2 of leiomyoma is a fibroid with more than $50 \%$ extension of its mass in the myometrium.

Type 3, 4 and 5 of leiomyomas are the intramural fibroids, which are completely within the wall of the uterus and due to their possible size, they may alter the uterine cavity or the shape of the uterus itself.

Type 6 and 7 are the sub serosal leiomyomas, they derive from the myometrium right at its external border and can have a pedunculated or broad base and also affect the neighboring structures due to direct pressure.

Type 8 fibroids are the cervical leiomyomas that, as their name suggests, are located in the cervix and not the uterine body [4]. 


\section{Discussion}

\section{Prevalence}

The uterine leiomyoma is the most frequently encountered benign tumor in the pelvic area of female patients. Its incidence is relatively difficult to assess accurately due to the lack of studies. In addition, the prevalence can be underestimated due to the fact that most studies were performed on symptomatic patients or those who underwent a hysterectomy procedure. Most studies have shown that between the ages of 25 and 44 the incidence of leiomyomas is proportional to the patient's age. Regarding the race, it was observed that the prevalence of uterine fibroids is higher in black patients compared to white females and has a rate of about 3 to 1 . In the general population, the incidence rate of uterine fibroids diagnosed by imagistic investigations or following a hysterectomy for other causes is about 9.2 per 1000 females overall [1-3,5].

\section{Risk factors and prevention}

There are a lot of external and internal factors that can influence the development of uterine leiomyoma. Early menarche (under 10 years of age), a poor diet, obesity, alcohol consumption (especially beer), the black race, essential hypertension and a familial predisposition are linked with a greater risk of fibroid genesis. On the other hand, factors like being physically active, having a healthy diet and having one or more pregnancies are all linked to having a lower risk of developing uterine fibroids [6-10].

\section{Clinical features}

Uterine fibroids are discovered incidentally on routine pelvic imaging or when they make the patient seek medical attention due to the symptomatology. Most of the uterine leiomyomas are asymptomatic due to their small size, but despite that many women encounter significant issues that affect their quality of life. The symptoms have a direct link with the size, number and location of the tumors. A myomatous uterus is described in size as a gravid one, more exactly in menstrual weeks $[11,12]$.

In practice there are four main symptoms that occur separately or grouped in a patient with uterine fibroids:

1. Heavy or prolonged menstrual bleeding

2. Bulk symptoms

3. Reproductive dysfunction

4. Pelvic pain (during the menses or outside them)

Usually, patients do not associate symptoms with uterine leiomyomas because they appear to be unrelated to the actual disease. Also, their onset is insidious and can cause the patient to attribute them to the aging process, or other problems unrelated to the real condition. Taking into account these things, we can say that patient education is important for detecting the onset of symptoms, which can be correlated with the imaging investigations or the physical examination. Finally, the size of the uterus can be helpful because many patients experience symptoms comparable with a pregnancy of 12 weeks gestational age [12-14].

\section{Diagnosis}

The diagnosis of uterine fibroids is generally established on pelvic examination and ultrasound examination which are often very suggestive. The symptoms that are not always present do nothing but suggest this diagnosis. The histopathological examination is performed only in cases where, a differential diagnosis with a neoplasm that has as a starting point the uterine tissue, is necessary [13].

During the consultation, the patient's anamnesis and her obstetrical and gynecological history are taken into account. Physical examination may reveal an enlarged uterus with an irregular surface. In the case of small submucosal or intramural fibroids, the pelvic examination may not show any change, at least at clinical evaluation. Symptoms such as heavy or prolonged menstrual bleeding, pelvic pain, or fertility problems may raise the suspicion of uterine fibroids and should be evaluated in detail $[13,14]$.

Pelvic ultrasound

Pelvic ultrasound is the investigation of choice in uterine leiomyomatosis and is the one that most often makes the diagnosis. Other investigations such as saline infusion ultrasonography, hysteroscopy or magnetic resonance imaging are used depending on the patient's clinical indications [13-15].

The first step of the paraclinical examination is the ultrasound examination which has a sensitivity of 95-100\% in highlighting the presence of fibroids in a uterus smaller in size than a 10 weeks of gestation uterus. The exact location of tumors becomes difficult in a larger uterus. Usually, the fibroids appear as hypoechoic formations, round-oval, well delimited and with a shadow cone.

The second step is to examine the uterine cavity in patients with suspected submucosal fibroids or who wish to preserve fertility. This can be examined using saline infusion ultrasonography that shows very well the submucosal and intramural fibroids that protrude into the uterine cavity. Another procedure that can be used for the same purpose is hysteroscopy. It can classify exactly the lesions that occupy a space in the uterine cavity, intracavitary formations, type 0 fibroids. It can also be useful to decide the type of treatment.

\section{Magnetic resonance imaging}

The last step of the paraclinical examination is magnetic resonance imaging. This investigation is indicated when the presence of a uterine neoplasm is suspected. It is the most efficient method to highlight the location and size of the lesions and can also distinguish between fibroids, adenomas and adenomyosis. Due to the 
high costs, it is indicated for patients who require complex surgeries. It is also useful before performing the uterine artery embolization procedure because MRI can approximate the results of this intervention [14-18].

\section{Treatment of submucosal fibroids}

In the past, the treatment of choice for submucosal fibroids was hysterectomy or abdominal myomectomy. These procedures have now been replaced by hysteroscopic myomectomy, a minimally invasive procedure that removes these lesions safely and effectively $[19,20]$. This intervention is used to remove intracavitary uterine fibroids and submucosal fibroids and some intramural fibroids. The advantages of hysteroscopic myomectomy are: the procedure can be performed on an outpatient basis, the recovery period is much shorter than a classic surgery, antalgic medication prescribed to the patient is minimal, postoperative restrictions imposed on the patient are minimal, post-procedure mortality is very low and uterine scar lesions are minimal.

\section{Indications}

The main indications for the hysteroscopic treatment of submucosal uterine fibroids are abnormal uterine bleeding, recurrent miscarriages and a history of infertility. At the same time, this intervention is also indicated in selected cases of patients with intracavitary fibroids that show symptoms that have as etiology other diseases besides uterine fibroids, such as: dysmenorrhea, leukorrhea, necrotic uterine fibroids after embolization, history of premature birth, postpartum hemorrhage, puerperal infections that have been aggravated by the presence of uterine fibroids and postmenopausal bleeding [21,22].

\section{Contraindications}

Contraindications to hysteroscopic myomectomy are: active pelvic infection, pyometra, intrauterine or implanted pregnancy in the cervix, uterine cancer. Other comorbidities of patients such as ischemic heart disease or bleeding diathesis may be relative contraindications, but being a minimally invasive intervention, it is rarely contraindicated [23].

Patients who are candidates for hysteroscopic myomectomy must also meet certain criteria related to the characteristics of uterine fibroids. The main types of fibroids approached by this technique are those with intracavitary evolution. Removal of fibroids that penetrate the myometrium, that are large or sessile can prolong the intervention and can promote the occurrence of perioperative complications. In the case of subserosally and intramural fibroids, hysteroscopic myomectomy cannot completely alleviate the symptoms. The uterine fibroids that can be successfully approached using this intervention are represented by type 0 , type I and type II classes, according to the classification of the European Hysteroscopy Society [24-26].
Observational studies have shown the effectiveness of the classification of the European Society of Hysteroscopy in predicting the total resection of fibroids, the results being the following: type 0 with efficacy between $96-97 \%$, type I with efficacy between $86-90 \%$ and type II with efficacy between $61-83 \%$. At the same time, the level of penetration in the myometrium is closely correlated with the volume of distended fluid absorbed. This feature was highlighted in a series of 339 hysteroscopic myomectomies in which it was observed that the volume of distended fluid absorbed increased the level of injury to the myometrium. The liquid values depending on the type of uterine fibroids are the following: type $0-450 \mathrm{ml}$, type I $-957 \mathrm{ml}$ and type II $1682 \mathrm{ml}$ [24-26].

\section{Complications}

Complications of this procedure are relatively rare, a report of more than 200 hysteroscopic myomectomies revealed a complication rate between 0.8 and $2.6 \%$. Another retrospective study of 235 interventions showed a different incidence rate of complications depending on the number of resected fibroids, $1.4 \%$ for resection of a single fibroid and $6.7 \%$ for multiple resections of fibroids [27,28].

The main complications occurred during the procedure are:

- uterine perforation: may occur in case of extensive resections.

- excessive absorption of distension fluid: it can also be found in massive resections of fibroids that also affect the myometrial component.

- excessive bleeding: usually blood loss is minimal, around $5-100 \mathrm{ml}$. When excessive bleeding occurs, it is mainly related to preoperative factors related to the patient or is attributed to uterine perforation.

- intrauterine adhesions: the appearance of this complication can generate fertility problems for the patient or problems related to menstruation. Retrospective studies have shown that the incidence rate of this complication varies from 0 to $46 \%$ and is higher as more fibroids are resected $[27,29,30]$.

The results of hysteroscopic myomectomies are quite difficult to assess because there is no consistency in studies on the types of resected fibroids, the degree of complications and the degree of recovery of patients both physically and fertility. At the same time, a series of studies highlight the high degree of satisfaction of patients after the procedure and the effective improvement of symptoms $[27,31]$.

Complete resection of fibroids depends on the degree of penetration of the myometrium, with incomplete resection rate ranging from 5 to $17 \%$. A study of 41 patients found that the 3-year reintervention rate for uterine fibroids was $44 \%$ [25,27]. The recurrence rate of uterine fibroids or abnormal uterine bleeding is approximately $20 \%$ among patients followed for 3 years postoperatively $[32,33]$. 
The chances of conception are improved in patients who have had a hysteroscopic myomectomy, however the effect on the rate of miscarriages is still unknown. (34)

Treatment of prolapsed pedunculated submucous leiomyoma

Uterine leiomyomas in statu nascendi are peduncled submucosal fibroids that due to uterine contractions are pushed through the cervix. These types of leiomyomas are usually resected by vaginal myomectomy. Removal of prolapsed pedunculated submucous leiomyoma is indicated in most patients, including asymptomatic ones because they may develop symptoms along the way. The intervention is performed either in an outpatient setting or in an operating room. The contraindications are few in number and are mainly represented by severe comorbidities of the patient [35].

Complications of vaginal myomectomy are rare, in a study of 46 patients no postoperative complications were observed. The most common complication is excessive bleeding at the site of fibroid implantation. If hemostasis cannot be performed in a timely manner, exploratory and curative hysteroscopy is indicated. Another complication that can occur is uterine inversion and is due to excessive traction force that is applied to the fibroid before it is resected. There is also a theoretical risk of infection but its frequency is unknown $[35,36]$.

The intervention is effective for most patients, with no reported cases of therapeutic failure except in situations where the fibroid pedicle is difficult to access. The recurrence of the prolapsed leiomyomas has a low frequency. A study of 46 patients who were followed for a period of 5 years showed that $9 \%$ of them underwent another vaginal myomectomy and 6\% underwent a hysterectomy that was indicated for causes other than fibroids. initially accused. In order to avoid the recurrence of the symptoms specific to uterine leiomyomas, hysteroscopy is indicated after a vaginal myomectomy [3537].

After the operation, patients may report uterine cramps and vaginal discharge or bleeding, symptoms that can last several days. The antalgic medication is usually in the minimum dose and the recovery is relatively fast [37].

\section{Conclusions}

Uterine fibromatosis is the most common benign tumor pathology in the uterus and causes bleeding outside the menstrual period, uterine deformity and conception problems. Submucosal fibroids are of 3 types and have mainly an indication for hysteroscopic myomectomy. Hysteroscopic myomectomy is indicated for resection of submucosal fibroids type 0, I and II. Hysteroscopic myomectomy is a minimally invasive intervention that is much more effective than conventional surgical treatments performed by abdominal approach. Hysteroscopic myomectomy has a low rate of postoperative complications and promotes rapid recovery of patients.

The degree of recurrence of fibromatosis is variable and is determined by the number of fibroids and the degree of interest of the myometrium. Accused fibroids are pediculated submucosal fibroids that proliferate through the cervix due to uterine contractility. The accused fibroids have an indication for resection even if the patients are asymptomatic.

Vaginal myomectomy is the chosen intervention for the treatment of fibroids. It is a minimally invasive procedure that can be performed in an outpatient setting or in an operating room. Vaginal myomectomy does not have a high rate of complications and the prognosis is favorable in most cases.

\section{Conflict of interest disclosure}

There are no known conflicts of interest in the publication of this article. The manuscript was read and approved by all authors.

\section{Compliance with ethical standards}

Any aspect of the work covered in this manuscript has been conducted with the ethical approval of all relevant bodies and that such approvals are acknowledged within the manuscript.

\section{References}

1. Baird DD, Dunson DB, Hill MC, Cousins D, Schectman JM. High cumulative incidence of uterine leiomyoma in black and white women: ultrasound evidence. Am J Obstet Gynecol. 2003 Jan;188(1):1007. doi: 10.1067/mob.2003.99

2. Serden SP, Brooks PG. Treatment of abnormal uterine bleeding with the gynecologic resectoscope. J Reprod Med. 1991 Oct;36(10):697-9.

3. Munro MG, Critchley HO, Fraser IS; FIGO Menstrual Disorders Working Group. The FIGO classification of causes of abnormal uterine bleeding in the reproductive years. Fertil Steril. 2011 Jun;95(7):2204-8, 2208.e1-3. doi: 10.1016/j.fertnstert.2011.03.079

4. Laughlin-Tommaso SK, Hesley GK, Hopkins MR, Brandt KR, Zhu Y, Stewart EA. Clinical limitations of the International Federation of Gynecology and Obstetrics (FIGO) classification of uterine fibroids. Int J Gynaecol Obstet. 2017 Nov;139(2):143-148. doi: 10.1002/ijgo.12266

5. Buttram VC Jr, Reiter RC. Uterine leiomyomata: etiology, symptomatology, and management. Fertil Steril. 1981 Oct;36(4):433-45. doi: 10.1016/s00150282(16)45789-4 
6. Ross RK, Pike MC, Vessey MP, Bull D, Yeates D, Casagrande JT. Risk factors for uterine fibroids: reduced risk associated with oral contraceptives. $\mathrm{Br}$ Med J (Clin Res Ed). 1986 Aug 9;293(6543):359-62. doi: 10.1136/bmj.293.6543.359

7. Parazzini F, La Vecchia C, Negri E, Cecchetti G, Fedele L. Epidemiologic characteristics of women with uterine fibroids: a case-control study. Obstet Gynecol. 1988 Dec;72(6):853-7. doi: 10.1097/00006250198812000-00008

8. Marshall LM, Spiegelman D, Goldman MB, Manson JE, Colditz GA, Barbieri RL, Stampfer MJ, Hunter DJ. A prospective study of reproductive factors and oral contraceptive use in relation to the risk of uterine leiomyomata. Fertil Steril. 1998 Sep;70(3):432-9. doi: 10.1016/s0015-0282(98)00208-8

9. Sarah Sghaier, Montassar Ghalleb, Hanen Bouaziz, Molka Chemlali, Monia Hechiche, Maher Slimane, Khaled Rahal. Sentinel lymphnode for edometrial cancer: where are we? J Clin Invest Surg. 2020;5(1):18. doi: 10.25083/2559.5555/5.1/1.8

10. Wise LA, Palmer JR, Harlow BL, Spiegelman D, Stewart EA, Adams-Campbell LL, Rosenberg L. Reproductive factors, hormonal contraception, and risk of uterine leiomyomata in African-American women: a prospective study. Am J Epidemiol. 2004 Jan 15;159(2):113-23. doi: 10.1093/aje/kwh016

11. Ghant MS, Sengoba KS, Recht H, Cameron KA, Lawson AK, Marsh EE. Beyond the physical: a qualitative assessment of the burden of symptomatic uterine fibroids on women's emotional and psychosocial health. J Psychosom Res. 2015; 78(5):499-503. doi: 10.1016/j.jpsychores.2014.12.016

12. Ghant MS, Sengoba KS, Vogelzang R, Lawson AK, Marsh EE. An Altered Perception of Normal: Understanding Causes for Treatment Delay in Women with Symptomatic Uterine Fibroids. $J$ Womens Health (Larchmt). 2016 Aug;25(8):846-52. doi: 10.1089/jwh.2015.5531

13. Monleón J, Cañete ML, Caballero V, Del Campo M, Doménech A, Losada MÁ, Calaf J; EME Study Group. Epidemiology of uterine myomas and clinical practice in Spain: An observational study. Eur J Obstet Gynecol Reprod Biol. 2018 Jul;226:59-65. doi: 10.1016/j.ejogrb.2018.05.026

14. Dueholm M, Lundorf E, Hansen ES, Ledertoug S, Olesen F. Accuracy of magnetic resonance imaging and transvaginal ultrasonography in the diagnosis, mapping, and measurement of uterine myomas. Am J Obstet Gynecol. 2002 Mar;186(3):409-15. doi: 10.1067/mob.2002.121725

15. Akış S, Kıran G, Göçmen A. The clinical importance of polyp size measurement through two-dimensional saline infusion sonohysterography prior to hysteroscopic resection in predicting premalignant and malignant endometrial lesions. Int J Gynaecol Obstet. 2021 Sep 12. doi: 10.1002/ijgo.13925

16. Cicinelli E, Romano F, Anastasio PS, Blasi N, Parisi C, Galantino P. Transabdominal sonohysterography, transvaginal sonography, and hysteroscopy in the evaluation of submucous myomas. Obstet Gynecol. 1995;85(1):42-7. doi: 10.1016/0029-7844(94)00298-r

17. Omary RA, Vasireddy S, Chrisman HB, Ryu RK, Pereles FS, Carr JC, Resnick SA, Nemcek AA Jr, Vogelzang RL. The effect of pelvic MR imaging on the diagnosis and treatment of women with presumed symptomatic uterine fibroids. J Vasc Interv Radiol. 2002 Nov;13(11):1149-53. doi: 10.1016/s10510443(07)61957-5

18. Vedantham S, Sterling KM, Goodwin SC, Spies JB, Shlansky-Goldberg R, Worthington-Kirsch RL, Andrews RT, Hovsepian DM, Smith SJ, Chrisman HB. I. Uterine fibroid embolization: preprocedure assessment. Tech Vasc Interv Radiol. 2002 Mar;5(1):216. doi: 10.1053/tvir.2002.124463

19. Wegienka G, Baird DD, Hertz-Picciotto I, Harlow SD, Steege JF, Hill MC, Schectman JM, Hartmann KE. Self-reported heavy bleeding associated with uterine leiomyomata. Obstet Gynecol. 2003 Mar;101(3):431-7. doi: 10.1016/s0029-7844(02)03121-6

20. Bohiltea R, Turcan N, Cavinder CM, Ducu I, Paunica I, Andronache LF, Cirstoiu MM. Risk factors, predictive markers and prevention strategies for intrauterine fetal death. An integrative review. J Mind Med Sci. 2020; 7(1):52-60. doi: 10.22543/7674.71.P5260

21. Akrivis Ch, Varras M, Bellou A, Kitsiou E, Stefanaki S, Antoniou N. Primary postpartum haemorrhage due to a large submucosal nonpedunculated uterine leiomyoma: a case report and review of the literature. Clin Exp Obstet Gynecol. 2003;30(2-3):156-8.

22. Mason TC, Adair J, Lee YC. Postpartum pyomyoma. $J$ Natl Med Assoc. 2005 Jun;97(6):826-8.

23. Hudspeth JC, Schwartz M, Fleming P, Ostrander T, Eyllon M. Essential Principles of Preoperative Assessment in Internal Medicine: A Case-Based Teaching Session. MedEdPORTAL. 2021 Aug 5;17:11178. doi: 10.15766/mep_2374-8265.11178

24. Wamsteker K, Emanuel MH, de Kruif JH. Transcervical hysteroscopic resection of submucous fibroids for abnormal uterine bleeding: results regarding the degree of intramural extension. Obstet Gynecol. 1993 Nov;82(5):736-40.

25. Van Dongen H, Emanuel MH, Smeets MJ, Trimbos B, Jansen FW. Follow-up after incomplete hysteroscopic removal of uterine fibroids. Acta Obstet Gynecol Scand. 2006;85(12):1463-7. doi: 10.1080/00016340600984647

26. Bacalbasa N, Balescu I, Dimitriu M, Balalau C, Vilcu M, Brezean I. Does sentinel lymph node detection play 
a role in patients with vaginal cancer? J Clin Invest Surg. 2019;4(1):1-4. doi: 10.25083/2559.5555/4.1/1.4.

27. Polena V, Mergui JL, Perrot N, Poncelet C, Barranger E, Uzan S. Long-term results of hysteroscopic myomectomy in 235 patients. Eur J Obstet Gynecol Reprod Biol. 2007 Feb;130(2):232-7. doi: 10.1016/j.ejogrb.2006.01.014

28. Jansen FW, Vredevoogd CB, van Ulzen K, Hermans J, Trimbos JB, Trimbos-Kemper TC. Complications of hysteroscopy: a prospective, multicenter study. Obstet Gynecol. 2000 Aug;96(2):266-70. doi: 10.1016/s00297844(00)00865-6

29. Taskin O, Sadik S, Onoglu A, Gokdeniz R, Erturan E, Burak F, Wheeler JM. Role of endometrial suppression on the frequency of intrauterine adhesions after resectoscopic surgery. J Am Assoc Gynecol Laparosc. $2000 \quad$ Aug;7(3):351-4. doi: 10.1016/s10743804(05)60478-1

30. Touboul C, Fernandez H, Deffieux X, Berry R, Frydman R, Gervaise A. Uterine synechiae after bipolar hysteroscopic resection of submucosal myomas in patients with infertility. Fertil Steril. 2009; 92(5):1690-3. doi: 10.1016/j.fertnstert.2008.08.108

31. Myers ER, Barber MD, Gustilo-Ashby T, Couchman G, Matchar DB, McCrory DC. Management of uterine leiomyomata: what do we really know? Obstet Gynecol. 2002 Jul;100(1):8-17. doi: 10.1016/s00297844(02)02019-7
32. Derman SG, Rehnstrom J, Neuwirth RS. The long-term effectiveness of hysteroscopic treatment of menorrhagia and leiomyomas. Obstet Gynecol. 1991 Apr;77(4):591-4.

33. Vercellini P, Zàina B, Yaylayan L, Pisacreta A, De Giorgi O, Crosignani PG. Hysteroscopic myomectomy: long-term effects on menstrual pattern and fertility. Obstet Gynecol. 1999 Sep;94(3):341-7. doi: 10.1016/s0029-7844(99)00346-4

34. Ubaldi F, Tournaye H, Camus M, Van der Pas H, Gepts E, Devroey P. Fertility after hysteroscopic myomectomy. Hum Reprod Update. 1995 Jan;1(1):8190. doi: 10.1093/humupd/1.1.81

35. Golan A, Zachalka N, Lurie S, Sagiv R, Glezerman M. Vaginal removal of prolapsed pedunculated submucous myoma: a short, simple, and definitive procedure with minimal morbidity. Arch Gynecol Obstet. 2005 Jan;271(1):11-3. doi: 10.1007/s00404-003-0590-x

36. Pană M, Sima RM, Bălălău OD, Stănescu AD, Pleş L, Poenaru MO. The quality of sexual life after vaginal surgical interventions. J Mind Med Sci. 2020;7(2):201205. doi: 10.22543/7674.72.P201205

37. Ben-Baruch G, Schiff E, Menashe Y, Menczer J. Immediate and late outcome of vaginal myomectomy for prolapsed pedunculated submucous myoma. Obstet Gynecol. 1988 Dec;72(6):858-61. doi: 10.1097/00006250-198812000-00009 